\title{
Gender Differences in Coffee Consumption and Its Effects in Young People
}

\author{
Shinichi Demura ${ }^{1}$, Hiroki Aoki ${ }^{2}$, Toshihide Mizusawa ${ }^{3}$, Kei Soukura ${ }^{3}$, Masahiro Noda $^{4}$, Toshiro Sato ${ }^{5}$ \\ ${ }^{1}$ Graduate School of Natural Science \& Technology, Kanazawa University, Kanazawa, Japan; ${ }^{2}$ Fukui National College of Technol- \\ ogy, Sabae, Japan; ${ }^{3}$ University of Fukui, Fukui, Japan; ${ }^{4}$ Jin-ai University, Echizen, Japan; ${ }^{5}$ Niigata University of Health and Welfare, \\ Niigata, Japan. \\ Email: aoki@fukui-nct.ac.jp
}

Received November $28^{\text {th }}, 2012$; revised January $5^{\text {th }}, 2013$; accepted January $14^{\text {th }}, 2013$

Copyright (c) 2013 Shinichi Demura et al. This is an open access article distributed under the Creative Commons Attribution License, which permits unrestricted use, distribution, and reproduction in any medium, provided the original work is properly cited.

\begin{abstract}
This study aimed to examine gender differences in coffee consumption and awareness of its effects in young people. To examine the above problem, a survey was conducted in 1189 young people (567 males aged $19.3 \pm 1.5$ years; 622 females aged $19.1 \pm 1.2$ years). The coffee consumption rate was significantly higher in males (50.8\%) than in females (32.8\%). In the coffee consumption group, no significant differences were found in the reasons for consumption, the components of coffee, and its effects on health. In the coffee nonconsumption group, significant gender differences were noted in the reasons for avoiding coffee; females (64.8\%) disliked its taste more than males (39.4\%). More young people of both genders in the consumption group were aware that coffee contains caffeine $(79.9 \%-86.5 \%)$ as compared with the nonconsumption group $(67.0 \%-74.2 \%)$. However, few people in both groups were aware about the components other than caffeine. In addition, more people in the nonconsumption group were unaware of the adverse effects of coffee on the body. The results of this study demonstrated that young males drink coffee more than young females. Among those who avoided coffee consumption, females disliked its taste more than males. Many people regardless of coffee consumption were aware about the components of coffee, but few knew about the other ingredients in this beverage. In addition, few people were aware of the negative effects of coffee on the health and body.
\end{abstract}

Keywords: Young; Males; Females; Coffee; Ingredients

\section{Introduction}

Coffee is a luxury drink that is widely consumed by people all over the world $(70 \%-80 \%)$ [1]. Coffee has a relaxing effect and it alleviates stress [2]. In addition, regular coffee drinking has been reported to reduce the risk of diabetes mellitus, hepatic cirrhosis, rectal cancer, cardiovascular disease, and other illnesses [3-9]. According to Garzaro et al. in 2011 [10], majority of the 100 subjects found the tested coffee-flavored iced dessert pleasant, on the basis of the Organoleptic-Sensorial Questionnaire (OSQ), in terms of taste, aspect, texture, and smell. No statistically significant differences were observed between the male and female subjects. Many people in Japan drink coffee regardless of age or gender [2]. However, gender differences in coffee consumption have not been adequately determined, although Yamazawa et al. [11] reported gender differences in coffee-flavored desserts and coffee consumption.

People drink coffee for different reasons, and these reasons may differ between males and females. Mirmiran et al. [12] reported gender as a factor influencing nutrient consumption. Du et al. [13] demonstrated that females have higher interest in the nutrients than males. Thus, knowledge about coffee may also differ between males and females.

Many studies have examined the effects of coffee consumption [5-9]. Information about the advantages and disadvantages of coffee consumption is easily available through the media. Compared with the nonconsumers, coffee consumers have more interest in and more knowledge about the various components in this beverage and its effects on the body. In addition, the sanative properties of coffee as a luxury drink have been the focus of one recent study [14]. However, coffee drinkers may have overly high expectations of the beneficial effects of coffee on health.

In this study, the effects of coffee consumption were examined in terms of gender differences in young people. 


\section{Methods}

1) Survey Method and Participants

A survey was administered to 1528 young people and data was gathered from subjects from a range of occupational fields, such as students, office workers, teachers, etc. Therefore, specific criteria for subject selection were not prepared. The survey (Table 1) was created with

Table 1. Survey items.

\begin{tabular}{|c|c|}
\hline Coffee ingestion (Yes or No) & \\
\hline \multicolumn{2}{|l|}{ Reasons of coffee consumption } \\
\hline \multirow{6}{*}{ Consumers } & 1. To wake up \\
\hline & 2. Relaxed \\
\hline & 3. Breath freshener \\
\hline & 4. Delicious \\
\hline & 5. Health-related \\
\hline & 6. Stress reduction \\
\hline \multicolumn{2}{|c|}{ Reasons of coffee nonconsumption } \\
\hline \multirow{4}{*}{ Nonconsumption } & 1. Stopped by doctor \\
\hline & 2. Dislike the taste \\
\hline & 3. Harmful for the body \\
\hline & 4. Expensive \\
\hline \multicolumn{2}{|c|}{ Ingredient included in coffee (Free answer) } \\
\hline \multicolumn{2}{|c|}{ Awareness on effects of coffee on health } \\
\hline \multirow{6}{*}{ Consumers } & 1. Nonexpectant \\
\hline & 2. Diabetes mellitus prevention \\
\hline & 3. Cancer prevention \\
\hline & 4. Diet \\
\hline & 5. Metabolic syndrome prevention \\
\hline & 6. Resolution of stress \\
\hline \multicolumn{2}{|c|}{ Awareness on effects of coffee to health } \\
\hline \multirow{6}{*}{ Nonconsumption } & 1. Unknown \\
\hline & 2. Diabetes mellitus prevention \\
\hline & 3. Cancer prevention \\
\hline & 4. Diet \\
\hline & 5. Metabolic syndrome prevention \\
\hline & 6. Resolution of stress \\
\hline \multicolumn{2}{|c|}{ Awareness on negative effects of coffee to body } \\
\hline \multirow{7}{*}{ Consumers and Nonconsumption } & 1. Unknown \\
\hline & 2. Stomach problems \\
\hline & 3. Close to a restroom \\
\hline & 4. Become to loose stools \\
\hline & 5. Dehydration \\
\hline & 6. Trembling hand \\
\hline & 7. Getting palpitations \\
\hline \multicolumn{2}{|c|}{ Consumption of drinks besides coffee } \\
\hline \multirow{9}{*}{ Consumers and Nonconsumption } & 1. Coffee drink \\
\hline & 2. Refreshing drinks with coffee \\
\hline & 3. Green tea \\
\hline & 4. Tea \\
\hline & 5. Juice \\
\hline & 6. Milk \\
\hline & 7. Water \\
\hline & 8. Carbonated drink \\
\hline & 9. Sports drink \\
\hline
\end{tabular}

reference to questionnaires from previous studies [2,15]. Survey items were selected on the basis of presence or absence of coffee consumption, reasons for coffee consumption or nonconsumption, ingredients in coffee, the effects of coffee on health, and preferred alternative drinks (see Table 1). Participants responded freely regarding the ingredients in coffee. Participants responded to questions included in the other survey items with a "yes" or "no."

2) Data Analysis

The chi-squared frequency test $\left(\chi^{2}\right.$ test) was used to clarify gender differences for each question item. The significance level in this study was set at $\mathrm{p}<0.05$, which was adjusted using the Bonferroni method.

\section{Results}

Validity testing determined that data for 1189 respondents (567 males aged $19.3 \pm 1.5$ years; 622 females aged $19.1 \pm 1.2$ years) were useful for the purposes of the analysis. Thus, the response rate was $86.9 \%$.The coffee consumption group comprised 288 males and 204 females, whereas the coffee nonconsumption group comprised 279 males and 418 females.

Table 2 shows the results for coffee consumption based on gender differences. The coffee consumption rate was significantly higher in males (50.8\%) than in females (32.8\%). Incidentally, coffee nonconsumption rate was significantly higher in females. Participants were grouped into those who drink coffee (coffee consumption group) and those who do not (coffee nonconsumption group).

Tables 3-7 show the reasons for coffee consumption (Table 3), ingredients in coffee (Table 4), and awareness of the positive (Table 5) and negative (Table 6) effects of coffee on health in the coffee consumption group. Consumption of drinks other than coffee (Table 7) with regard to gender differences in the coffee consumption group are shown in Table 7. Response rates in males and females were similar $(1.7 \%$ - $46.2 \%$ vs. $0.0 \%$ - 46.1\%) for all items regarding reasons for consumption. Hence, no significant gender differences were found in responses to these items, although approximately 50\% respondents answered that coffee helped them to wake up or that they considered it delicious. Most respondents of both genders (approximately 80\%) knew that coffee contains caffeine, but few were aware of other ingredients in coffee $(4.9 \%$ $5.4 \%$ ). No significant gender differences were noted regarding the effects of coffee on health; males and females responded similarly $(1.0 \%-85.1 \%$ vs. $1.0 \%$ $83.3 \%$ ) for all items in this category. More than their 80\% answered to "not expectation" for the above-stated in particular. Similar response rates were identified for males and females $(2.8 \%-42.0 \%$ vs. $2.0 \%$ - 40.7\%) for all items regarding the negative effects of coffee on health; 
Table 2. Coffee consumption by gender.

\begin{tabular}{ccccccc}
\hline & \multicolumn{2}{c}{ Males $(\mathrm{n}=567)$} & \multicolumn{2}{c}{ Females $(\mathrm{n}=622)$} & $\chi^{2}$ & $\mathrm{p}$ \\
\cline { 2 - 5 } & $\mathrm{n}$ & & $\mathrm{n}$ & & \\
\hline Coffee consumption & 288 & $(50.8 \%)$ & 204 & $(32.8 \%)$ & $39.6^{*}$ & 0.00 \\
$\quad$ Coffee nonconsumption & 279 & $(49.2 \%)$ & 418 & $(67.2 \%)$ & & \\
${ }^{*} \mathrm{p}<0.05$. & & & & &
\end{tabular}

Table 3. Gender differences regarding reasons for consuming coffee.

\begin{tabular}{|c|c|c|c|c|c|c|c|}
\hline & & \multicolumn{2}{|c|}{ Males (n = 288) } & \multicolumn{2}{|c|}{ Females $(\mathrm{n}=204)$} & \multirow{2}{*}{$\chi^{2}$} & \multirow{2}{*}{$\mathrm{p}$} \\
\hline & & $\mathrm{n}$ & & $\mathrm{n}$ & & & \\
\hline \multirow{2}{*}{ 1. To wake up } & Yes & 133 & $(46.2 \%)$ & 94 & $(46.1 \%)$ & \multirow{2}{*}{0.00} & \multirow{2}{*}{0.98} \\
\hline & No & 155 & $(53.8 \%)$ & 110 & (53.9\%) & & \\
\hline \multirow{2}{*}{ 2. Relaxed } & Yes & 104 & (36.1\%) & 61 & (29.9\%) & \multirow{2}{*}{2.07} & \multirow{2}{*}{0.15} \\
\hline & No & 184 & (63.9\%) & 143 & $(70.1 \%)$ & & \\
\hline \multirow{2}{*}{ 3. Breath freshener } & Yes & 106 & $(36.8 \%)$ & 61 & $(29.9 \%)$ & \multirow{2}{*}{2.54} & \multirow{2}{*}{0.11} \\
\hline & No & 182 & $(63.2 \%)$ & 143 & $(70.1 \%)$ & & \\
\hline \multirow{2}{*}{ 4. Delicious } & Yes & 133 & $(46.2 \%)$ & 94 & $(46.1 \%)$ & \multirow{2}{*}{0.00} & \multirow{2}{*}{0.98} \\
\hline & No & 155 & (53.8\%) & 110 & (53.9\%) & & \\
\hline \multirow{2}{*}{ 5. Health-related } & Yes & 5 & $(1.7 \%)$ & 0 & $(0.0 \%)$ & \multirow{2}{*}{3.58} & \multirow{2}{*}{0.06} \\
\hline & No & 283 & (98.3\%) & 204 & $(100.0 \%)$ & & \\
\hline \multirow{2}{*}{ 6. Stress reduction } & Yes & 11 & $(3.8 \%)$ & 4 & $(2.0 \%)$ & \multirow{2}{*}{1.40} & \multirow{2}{*}{0.24} \\
\hline & No & 277 & (96.2\%) & 200 & (98.0\%) & & \\
\hline
\end{tabular}

Table 4. Gender differences regarding awareness of components in the coffee consumption group.

\begin{tabular}{|c|c|c|c|c|c|c|c|}
\hline & & \multicolumn{2}{|c|}{ Males $(n=288)$} & \multicolumn{2}{|c|}{ Females $(n=204)$} & \multirow{2}{*}{$\chi^{2}$} & \multirow{2}{*}{$\mathrm{p}$} \\
\hline & & $\mathrm{n}$ & & $\mathrm{n}$ & & & \\
\hline \multirow{2}{*}{ 1. Caffeine } & Yes & 249 & (86.5\%) & 163 & (79.9\%) & \multirow{2}{*}{3.77} & \multirow{2}{*}{0.05} \\
\hline & No & 39 & $(13.5 \%)$ & 41 & (20.1\%) & & \\
\hline \multirow{2}{*}{ 2. Polyphenol } & Yes & 14 & $(4.9 \%)$ & 11 & $(5.4 \%)$ & \multirow{2}{*}{0.07} & \multirow{2}{*}{0.79} \\
\hline & No & 274 & (95.1\%) & 193 & (94.6\%) & & \\
\hline
\end{tabular}

Table 5. Gender differences regarding awareness of the positive effects of coffee on health in the coffee consumption group.

\begin{tabular}{|c|c|c|c|c|c|c|c|}
\hline & & \multicolumn{2}{|c|}{ Males $(\mathrm{n}=288)$} & \multicolumn{2}{|c|}{ Females $(\mathrm{n}=204)$} & \multirow{2}{*}{$\chi^{2}$} & \multirow{2}{*}{$\mathrm{p}$} \\
\hline & & $\mathrm{n}$ & & $\mathrm{n}$ & & & \\
\hline \multirow{2}{*}{ 1. Nonexpectant } & Yes & 245 & $(85.1 \%)$ & 170 & $(83.3 \%)$ & \multirow{2}{*}{0.27} & \multirow{2}{*}{0.60} \\
\hline & No & 43 & $(14.9 \%)$ & 34 & $(16.7 \%)$ & & \\
\hline \multirow{2}{*}{ 2. Diabetes mellitus prevention } & Yes & 3 & $(1.0 \%)$ & 2 & $(1.0 \%)$ & \multirow{2}{*}{0.00} & \multirow{2}{*}{0.95} \\
\hline & No & 285 & $(99.0 \%)$ & 202 & $(99.0 \%)$ & & \\
\hline \multirow{2}{*}{ 3. Cancer prevention } & Yes & 4 & $(1.4 \%)$ & 5 & $(2.5 \%)$ & \multirow{2}{*}{0.75} & \multirow{2}{*}{0.35} \\
\hline & No & 284 & (98.6\%) & 199 & $(97.5 \%)$ & & \\
\hline \multirow{2}{*}{ 4. Diet } & Yes & 7 & $(2.4 \%)$ & 4 & $(2.0 \%)$ & \multirow{2}{*}{0.12} & \multirow{2}{*}{0.73} \\
\hline & No & 281 & $(97.6 \%)$ & 200 & $(98.0 \%)$ & & \\
\hline \multirow{2}{*}{ 5. Metabolic syndrome prevention } & Yes & 5 & $(1.7 \%)$ & 1 & $(0.5 \%)$ & \multirow{2}{*}{1.54} & \multirow{2}{*}{0.22} \\
\hline & No & 283 & (98.3\%) & 203 & (99.5\%) & & \\
\hline \multirow{2}{*}{ 6. Resolution of stress } & Yes & 31 & $(10.8 \%)$ & 19 & $(9.3 \%)$ & \multirow{2}{*}{0.28} & \multirow{2}{*}{0.60} \\
\hline & No & 257 & $(89.2 \%)$ & 185 & $(90.7 \%)$ & & \\
\hline
\end{tabular}

no significant gender differences were found for this parameter. Approximately $40 \%$ respondents of both genders answered that they were unaware of any negative effects of coffee or that they found the need to be close to a restroom after drinking coffee. For questions regarding alternative beverages, significant gender differences were 
Table 6. Gender differences regarding awareness of the negative effects of coffee on the body in the coffee consumption group.

\begin{tabular}{|c|c|c|c|c|c|c|c|}
\hline & & \multicolumn{2}{|c|}{ Males $(\mathrm{n}=288)$} & \multicolumn{2}{|c|}{ Females $(\mathrm{n}=204)$} & \multirow{2}{*}{$\chi^{2}$} & \multirow{2}{*}{$\mathrm{p}$} \\
\hline & & $\mathrm{n}$ & & $\mathrm{n}$ & & & \\
\hline \multirow{2}{*}{ 1. Unknown } & Yes & 121 & $(42.0 \%)$ & 74 & (36.3\%) & \multirow{2}{*}{1.64} & \multirow{2}{*}{0.20} \\
\hline & No & 167 & $(58.0 \%)$ & 130 & $(63.7 \%)$ & & \\
\hline \multirow{2}{*}{ 2. Stomach problems } & Yes & 70 & $(24.3 \%)$ & 57 & $(27.9 \%)$ & \multirow{2}{*}{0.82} & \multirow{2}{*}{0.36} \\
\hline & No & 218 & $(75.7 \%)$ & 147 & $(72.1 \%)$ & & \\
\hline \multirow{2}{*}{ 3. Close to a restroom } & Yes & 106 & (36.8\%) & 83 & (40.7\%) & \multirow{2}{*}{0.76} & \multirow{2}{*}{0.38} \\
\hline & No & 182 & $(63.2 \%)$ & 121 & $(59.3 \%)$ & & \\
\hline \multirow{2}{*}{ 4. Become to loose stools } & Yes & 30 & $(10.4 \%)$ & 27 & $(13.2 \%)$ & \multirow{2}{*}{0.93} & \multirow{2}{*}{0.34} \\
\hline & No & 258 & (89.6\%) & 177 & $(86.8 \%)$ & & \\
\hline \multirow{2}{*}{ 5. Dehydration } & Yes & 12 & $(4.2 \%)$ & 8 & $(3.9 \%)$ & \multirow{2}{*}{0.02} & \multirow{2}{*}{0.89} \\
\hline & No & 276 & $(95.8 \%)$ & 196 & (96.1\%) & & \\
\hline \multirow{2}{*}{ 6. Trembling hand } & Yes & 8 & $(2.8 \%)$ & 4 & $(2.0 \%)$ & \multirow{2}{*}{0.34} & \multirow{2}{*}{0.56} \\
\hline & No & 280 & $(97.2 \%)$ & 200 & $(98.0 \%)$ & & \\
\hline \multirow{2}{*}{ 7. Getting palpitations } & Yes & 15 & $(5.2 \%)$ & 2 & $(1.0 \%)$ & \multirow{2}{*}{6.40} & \multirow{2}{*}{0.01} \\
\hline & No & 273 & (94.8\%) & 202 & (99.0\%) & & \\
\hline
\end{tabular}

Table 7. Gender differences regarding consumption of other drinks in the coffee consumption group.

\begin{tabular}{|c|c|c|c|c|c|c|c|}
\hline & & \multicolumn{2}{|c|}{ Males $(\mathrm{n}=288)$} & \multicolumn{2}{|c|}{ Females $(n=204)$} & \multirow{2}{*}{$\chi^{2}$} & \multirow{2}{*}{$\mathrm{p}$} \\
\hline & & $\mathrm{n}$ & & $\mathrm{n}$ & & & \\
\hline \multirow{2}{*}{ 1. Coffee drink } & Yes & 37 & $(12.8 \%)$ & 30 & $(14.7 \%)$ & \multirow{2}{*}{0.35} & \multirow{2}{*}{0.55} \\
\hline & No & 251 & (87.2\%) & 174 & $(85.3 \%)$ & & \\
\hline \multirow{2}{*}{ 2. Refreshing drinks with coffee } & Yes & 25 & $(8.7 \%)$ & 15 & $(7.4 \%)$ & \multirow{2}{*}{0.28} & \multirow{2}{*}{0.60} \\
\hline & No & 263 & (91.3\%) & 189 & $(92.6 \%)$ & & \\
\hline \multirow{2}{*}{ 3. Green tea } & Yes & 162 & $(56.3 \%)$ & 134 & $(65.7 \%)$ & \multirow{2}{*}{4.44} & \multirow{2}{*}{0.04} \\
\hline & No & 126 & $(43.8 \%)$ & 70 & $(34.3 \%)$ & & \\
\hline \multirow{2}{*}{ 4. Tea } & Yes & 59 & $(20.5 \%)$ & 77 & $(37.7 \%)$ & \multirow{2}{*}{$17.78^{*}$} & \multirow{2}{*}{0.00} \\
\hline & No & 229 & (79.5\%) & 127 & $(62.3 \%)$ & & \\
\hline \multirow{2}{*}{ 5. Juice } & Yes & 86 & $(29.9 \%)$ & 50 & $(24.5 \%)$ & \multirow{2}{*}{1.71} & \multirow{2}{*}{0.19} \\
\hline & No & 202 & $(70.1 \%)$ & 154 & $(75.5 \%)$ & & \\
\hline \multirow{2}{*}{ 6. Milk } & Yes & 89 & (30.9\%) & 49 & $(24.0 \%)$ & \multirow{2}{*}{2.80} & \multirow{2}{*}{0.09} \\
\hline & No & 199 & $(69.1 \%)$ & 155 & $(76.0 \%)$ & & \\
\hline \multirow{2}{*}{ 7. Water } & Yes & 103 & (35.8\%) & 72 & (35.3\%) & \multirow{2}{*}{0.11} & \multirow{2}{*}{0.92} \\
\hline & No & 185 & $(64.2 \%)$ & 132 & $(64.7 \%)$ & & \\
\hline \multirow{2}{*}{ 8. Carbonated drink } & Yes & 84 & $(29.2 \%)$ & 32 & $(15.7 \%)$ & \multirow{2}{*}{$12.04^{*}$} & \multirow{2}{*}{0.00} \\
\hline & No & 204 & (70.8\%) & 172 & (84.3\%) & & \\
\hline \multirow{2}{*}{ 9. Sports drink } & Yes & 69 & $(24.0 \%)$ & 16 & $(7.8 \%)$ & \multirow{2}{*}{$21.70^{*}$} & \multirow{2}{*}{0.00} \\
\hline & No & 219 & (76.0\%) & 188 & (92.2\%) & & \\
\hline
\end{tabular}

${ }^{*} \mathrm{p}<0.05 / 9=0.0056$.

found in the percentage of drinkers of tea (20.5\% vs. $37.7 \%$ ), carbonated drinks ( $29.2 \%$ vs. $15.7 \%$ ), and sports drinks (24.0\% vs. $7.8 \%)$. Female respondents consumed tea $(20.5 \%$ vs. $37.7 \%)$ more than males, whereas males consumed more carbonated drinks and sports drinks than females.

Tables 8-12 show the reasons for coffee nonconsumption (Table 8), components of coffee (Table 9), and awareness of the positive (Table 10) and negative (Table
11) effects of coffee on health in the coffee nonconsumption group. Consumption of drinks other than coffee in terms of gender differences in the coffee nonconsumption group are shown in Table 12. Significant gender differences were found for the survey items regarding the taste and cost of coffee. More females disliked the taste of coffee (64.8\%) than males (39.4\%), but responses to the latter survey item were extremely few $(0.2 \%-5 \%)$. Significant gender differences were found in the aware- 
Table 8. Gender differences regarding reasons for coffee nonconsumption.

\begin{tabular}{|c|c|c|c|c|c|c|c|}
\hline & & \multicolumn{2}{|c|}{ Males $(n=279)$} & \multicolumn{2}{|c|}{ Females $(n=418)$} & \multirow{2}{*}{$\chi^{2}$} & \multirow{2}{*}{$\mathrm{p}$} \\
\hline & & $\mathrm{n}$ & & $\mathrm{n}$ & & & \\
\hline \multirow{2}{*}{ 1. Stopped by doctor } & Yes & 1 & $(0.4 \%)$ & 1 & $(0.2 \%)$ & \multirow{2}{*}{0.08} & \multirow{2}{*}{0.77} \\
\hline & No & 278 & (99.6\%) & 417 & (99.8\%) & & \\
\hline \multirow{2}{*}{ 2. Dislike the taste } & Yes & 110 & (39.4\%) & 271 & (64.8\%) & \multirow{2}{*}{$43.58^{*}$} & \multirow{2}{*}{0.00} \\
\hline & No & 169 & $(60.6 \%)$ & 147 & (35.2\%) & & \\
\hline \multirow{2}{*}{ 3. Harmful for the body } & Yes & 9 & $(3.2 \%)$ & 10 & $(2.4 \%)$ & \multirow{2}{*}{0.44} & \multirow{2}{*}{0.51} \\
\hline & No & 270 & (96.8\%) & 408 & $(97.6 \%)$ & & \\
\hline \multirow{2}{*}{ 4. Expensive } & Yes & 14 & $(5.0 \%)$ & 1 & $(0.2 \%)$ & \multirow{2}{*}{$18.15^{*}$} & \multirow{2}{*}{0.00} \\
\hline & No & 265 & (95.0\%) & 417 & (99.8\%) & & \\
\hline
\end{tabular}

${ }^{*} \mathrm{p}<0.05 / 4=0.0125$.

Table 9. Gender differences regarding awareness of ingredients in the coffee nonconsumption group.

\begin{tabular}{|c|c|c|c|c|c|c|c|}
\hline & & \multicolumn{2}{|c|}{ Males $(\mathrm{n}=279)$} & \multicolumn{2}{|c|}{ Females $(\mathrm{n}=418)$} & \multirow{2}{*}{$\chi^{2}$} & \multirow{2}{*}{$\mathrm{p}$} \\
\hline & & $\mathrm{n}$ & & $\mathrm{n}$ & & & \\
\hline \multirow{2}{*}{ 1. Caffeine } & Yes & 207 & $(74.2 \%)$ & 280 & $(67.0 \%)$ & \multirow{2}{*}{4.13} & \multirow{2}{*}{0.04} \\
\hline & No & 72 & $(25.8 \%)$ & 138 & $(33.0 \%)$ & & \\
\hline \multirow{2}{*}{ 2. Polyphenol } & Yes & 9 & $(3.2 \%)$ & 33 & $(7.9 \%)$ & \multirow{2}{*}{$6.44^{*}$} & \multirow{2}{*}{0.01} \\
\hline & No & 270 & (96.8\%) & 385 & $(92.1 \%)$ & & \\
\hline
\end{tabular}

${ }^{*} \mathrm{p}<0.05 / 2=0.025$.

Table 10. Gender differences regarding awareness of the positive effects of coffee on health in the coffee nonconsumption group.

\begin{tabular}{|c|c|c|c|c|c|c|c|}
\hline & & \multicolumn{2}{|c|}{ Males $(n=279)$} & \multicolumn{2}{|c|}{ Females $(n=418)$} & \multirow{2}{*}{$\chi^{2}$} & \multirow{2}{*}{$\mathrm{p}$} \\
\hline & & $\mathrm{n}$ & & $\mathrm{n}$ & & & \\
\hline \multirow{2}{*}{ 1. Unknown } & Yes & 224 & (80.3\%) & 332 & (79.4\%) & \multirow{2}{*}{0.08} & \multirow{2}{*}{0.78} \\
\hline & No & 55 & $(19.7 \%)$ & 86 & (20.6\%) & & \\
\hline \multirow{2}{*}{ 2. Diabetes mellitus prevention } & Yes & 2 & $(0.7 \%)$ & 2 & $(0.5 \%)$ & \multirow{2}{*}{0.17} & \multirow{2}{*}{0.68} \\
\hline & No & 277 & (99.3\%) & 416 & (99.5\%) & & \\
\hline \multirow{2}{*}{ 3. Cancer prevention } & Yes & 8 & (2.9\%) & 13 & (3.1\%) & \multirow{2}{*}{0.03} & \multirow{2}{*}{0.85} \\
\hline & No & 271 & $(97.1 \%)$ & 405 & (96.9\%) & & \\
\hline \multirow{2}{*}{ 4. Diet } & Yes & 5 & $(1.8 \%)$ & 23 & $(5.5 \%)$ & \multirow{2}{*}{5.97} & \multirow{2}{*}{0.02} \\
\hline & No & 274 & $(98.2 \%)$ & 395 & (94.5\%) & & \\
\hline \multirow{2}{*}{ 5. Metabolic syndrome prevention } & Yes & 3 & $(1.1 \%)$ & 7 & $(1.7 \%)$ & \multirow{2}{*}{0.43} & \multirow{2}{*}{0.51} \\
\hline & No & 276 & (98.9\%) & 411 & (98.3\%) & & \\
\hline \multirow{2}{*}{ 6. Resolution of stress } & Yes & 32 & $(11.5 \%)$ & 27 & $(6.5 \%)$ & \multirow{2}{*}{5.42} & \multirow{2}{*}{0.02} \\
\hline & No & 247 & (88.5\%) & 391 & (93.5\%) & & \\
\hline
\end{tabular}

Table 11. Gender differences regarding awareness of the negative effects of coffee on the body in the coffee nonconsumption group.

\begin{tabular}{|c|c|c|c|c|c|c|c|}
\hline & & \multicolumn{2}{|c|}{ Males $(\mathrm{n}=279)$} & \multicolumn{2}{|c|}{ Females $(\mathrm{n}=418)$} & \multirow{2}{*}{$\chi^{2}$} & \multirow{2}{*}{$\mathrm{p}$} \\
\hline & & $\mathrm{n}$ & & $\mathrm{n}$ & & & \\
\hline \multirow{2}{*}{ 1. Unknown } & Yes & 173 & (62.0\%) & 234 & $(56.0 \%)$ & \multirow{2}{*}{2.50} & \multirow{2}{*}{0.11} \\
\hline & No & 106 & $(38.0 \%)$ & 184 & $(44.0 \%)$ & & \\
\hline \multirow{2}{*}{ 2. Stomach problems } & Yes & 43 & $(15.4 \%)$ & 66 & $(15.8 \%)$ & \multirow{2}{*}{0.02} & \multirow{2}{*}{0.89} \\
\hline & No & 236 & $(84.6 \%)$ & 352 & (84.2\%) & & \\
\hline \multirow{2}{*}{ 3. Close to a restroom } & Yes & 58 & (20.8\%) & 97 & $(23.2 \%)$ & \multirow{2}{*}{0.57} & \multirow{2}{*}{0.45} \\
\hline & No & 221 & $(79.2 \%)$ & 321 & $(76.8 \%)$ & & \\
\hline \multirow{2}{*}{4 .Become to loose stools } & Yes & 19 & $(6.8 \%)$ & 30 & $(7.2 \%)$ & \multirow{2}{*}{0.03} & \multirow{2}{*}{0.85} \\
\hline & No & 260 & $(93.2 \%)$ & 388 & $(92.8 \%)$ & & \\
\hline \multirow{2}{*}{ 5. Dehydration } & Yes & 2 & $(0.7 \%)$ & 9 & $(2.2 \%)$ & \multirow{2}{*}{2.22} & \multirow{2}{*}{0.14} \\
\hline & No & 277 & (99.3\%) & 409 & $(97.8 \%)$ & & \\
\hline \multirow{2}{*}{ 6. Trembling hand } & Yes & 4 & $(1.4 \%)$ & 7 & $(1.7 \%)$ & \multirow{2}{*}{0.06} & \multirow{2}{*}{0.80} \\
\hline & No & 275 & $(98.6 \%)$ & 411 & (98.3\%) & & \\
\hline \multirow{2}{*}{ 7. Getting palpitations } & Yes & 4 & $(1.4 \%)$ & 6 & $(1.4 \%)$ & \multirow{2}{*}{0.06} & \multirow{2}{*}{0.80} \\
\hline & No & 275 & $(98.6 \%)$ & 412 & (98.6\%) & & \\
\hline
\end{tabular}


Table 12. Gender differences regarding consumption of drinks other than coffee in the coffee nonconsumption group.

\begin{tabular}{|c|c|c|c|c|c|c|c|}
\hline & & \multicolumn{2}{|c|}{ Males $(n=279)$} & \multicolumn{2}{|c|}{ Females $(n=418)$} & \multirow{2}{*}{$\chi^{2}$} & \multirow{2}{*}{$\mathrm{p}$} \\
\hline & & $\mathrm{n}$ & & $\mathrm{n}$ & & & \\
\hline \multirow{2}{*}{ 1. Coffee drink } & Yes & 18 & $(6.5 \%)$ & 36 & $(8.6 \%)$ & \multirow{2}{*}{1.09} & \multirow{2}{*}{0.30} \\
\hline & No & 261 & (93.5\%) & 382 & $(91.4 \%)$ & & \\
\hline \multirow{2}{*}{ 2. Refreshing drinks with coffee } & Yes & 17 & $(6.1 \%)$ & 34 & $(8.1 \%)$ & \multirow{2}{*}{1.03} & \multirow{2}{*}{0.31} \\
\hline & No & 262 & (93.9\%) & 384 & $(91.9 \%)$ & & \\
\hline \multirow{2}{*}{ 3. Green tea } & Yes & 180 & $(64.5 \%)$ & 325 & $(77.8 \%)$ & \multirow{2}{*}{$14.69^{*}$} & \multirow{2}{*}{0.00} \\
\hline & No & 99 & $(35.5 \%)$ & 93 & $(22.2 \%)$ & & \\
\hline \multirow{2}{*}{ 4. Tea } & Yes & 42 & $(15.1 \%)$ & 121 & (28.9\%) & \multirow{2}{*}{$18.03^{*}$} & \multirow{2}{*}{0.00} \\
\hline & No & 237 & $(84.9 \%)$ & 297 & $(71.1 \%)$ & & \\
\hline \multirow{2}{*}{ 5. Juice } & Yes & 114 & $(40.9 \%)$ & 156 & $(37.3 \%)$ & \multirow{2}{*}{0.88} & \multirow{2}{*}{0.35} \\
\hline & No & 165 & $(59.1 \%)$ & 262 & $(62.7 \%)$ & & \\
\hline \multirow{2}{*}{ 6. Milk } & Yes & 95 & (34.1\%) & 108 & $(25.8 \%)$ & \multirow{2}{*}{5.47} & \multirow{2}{*}{0.02} \\
\hline & No & 184 & $(65.9 \%)$ & 310 & $(74.2 \%)$ & & \\
\hline \multirow{2}{*}{ 7. Water } & Yes & 127 & $(45.5 \%)$ & 207 & $(49.5 \%)$ & \multirow{2}{*}{1.07} & \multirow{2}{*}{0.30} \\
\hline & No & 152 & $(54.5 \%)$ & 211 & (50.5\%) & & \\
\hline \multirow{2}{*}{ 8. Carbonated drink } & Yes & 81 & (29.0\%) & 78 & (18.7\%) & \multirow{2}{*}{$10.22^{*}$} & \multirow{2}{*}{0.00} \\
\hline & No & 198 & $(71.0 \%)$ & 340 & (81.3\%) & & \\
\hline \multirow{2}{*}{ 9. Sports drink } & Yes & 79 & $(28.3 \%)$ & 74 & $(17.7 \%)$ & \multirow{2}{*}{$11.00^{*}$} & \multirow{2}{*}{0.00} \\
\hline & No & 200 & (71.7\%) & 344 & $(82.3 \%)$ & & \\
\hline
\end{tabular}

${ }^{*} \mathrm{p}<0.05 / 9=0.0056$.

ness of polyphenol as a component of coffee; however, the response rate for this survey item was extremely low (3\% - 8\%). Most males and females (approximately 70\%) knew that coffee contains caffeine. No significant gender differences were found in awareness of the effects of coffee on health; responses from males and females were similar $(1.1 \%-80.3 \%$ vs. $1.7 \%-79.4 \%)$ for all items. More than their $80 \%$ answered that they were unaware of the positive effects of coffee on health. Answer rates for males and females were similar $(0.7 \%-62.0 \%$ vs. $1.4 \%$ $56.0 \%$ ) for all items regarding the negative effects of coffee on health, so no significant gender differences were found for these items. Approximately 50\% males and females answered that they were unaware of the negative effects of coffee on health. For questions regarding alternative beverages, significant gender differences were found in the percentage of drinkers of green tea $(64.5 \%$ vs. $77.8 \%)$ and tea $(15.1 \%$ vs. $28.9 \%)$, carbonated drinks $(29.0 \%$ vs. $18.7 \%)$, and sports drinks (28.3\% vs. $17.7 \%)$. Females consumed more tea than males $(20.5 \%$ vs. $37.7 \%)$, and males consumed more carbonated drinks and sports drinks in the coffee nonconsumption group.

Tables 13-15 show gender differences regarding awareness of ingredients (Table 13) and awareness of the positive (Table 14) and negative (Table 15) effects of coffee on health and the body between the coffee consumption and nonconsumption groups. A significant group difference was found only for caffeine. More respondents in the consumption group were aware of the effects of caffeine, but more than $65 \%$ respondents in the nonconsumption group also were aware of this fact. No significant differences were found in the awareness of the effects of coffee on health between both groups $(1.1 \%$ $10.8 \%$ for the consumption group vs. $0.5 \%-9.3 \%$ for the nonconsumption group). No significant group differences were found in responses indicating lack of awareness of negative effects of coffee on health and need to be close to a restroom. More respondents in the nonconsumption group ( $>50 \%$ ) were unaware of the negative effects of coffee on health, and more respondents in the consumption group (approximately 40\%) indicated the need to be close to a restroom after drinking coffee. In addition, more respondents in the consumption group reported stomach problems due to coffee consumption (27.9\% vs. $15.8 \%)$.

\section{Discussion}

The present study results indicated that more young males (50.8\%) drank coffee than young females (32.8\%). According to Yamazawa et al. [11], gender differences in preferences for sweet coffee were related to coffee drinking habits. Rodenburg et al. [16], comparing coffee consumption among 4 groups of smoking and nonsmoking males and females, found that nonsmoking females consumed less coffee. The present study result indicated that females drank Japanese tea or green tea more often than males in both the coffee consumption and nonconsump- 
Table 13. Gender differences between the consumption and nonconsumption groups in terms of awareness of ingredients in coffee.

\begin{tabular}{|c|c|c|c|c|c|c|c|c|}
\hline & & & \multicolumn{2}{|c|}{ Consumption } & \multicolumn{2}{|c|}{ Nonconsumption } & \multirow{2}{*}{$\chi^{2}$} & \multirow{2}{*}{$\mathrm{p}$} \\
\hline & & & $\mathrm{n}$ & $\%$ & $\mathrm{n}$ & $\%$ & & \\
\hline \multirow{4}{*}{ Males } & \multirow{2}{*}{ 1. Caffeine } & Yes & 249 & (86.5\%) & 207 & $(74.2 \%)$ & \multirow{2}{*}{$13.5^{*}$} & \multirow{2}{*}{0.00} \\
\hline & & No & 39 & (13.5\%) & 72 & $(25.8 \%)$ & & \\
\hline & \multirow{2}{*}{ 2. Polyphenol } & Yes & 14 & $(4.9 \%)$ & 9 & (3.2\%) & \multirow{2}{*}{0.97} & \multirow{2}{*}{0.32} \\
\hline & & No & 274 & (95.1\%) & 270 & (96.8\%) & & \\
\hline \multirow{4}{*}{ Females } & \multirow{2}{*}{ 1. Caffeine } & Yes & 163 & (79.9\%) & 280 & $(67.0 \%)$ & \multirow{2}{*}{$11.2^{*}$} & \multirow{2}{*}{0.00} \\
\hline & & No & 41 & (20.1\%) & 138 & (33.0\%) & & \\
\hline & \multirow{2}{*}{ 2. Polyphenol } & Yes & 11 & $(5.4 \%)$ & 33 & $(7.9 \%)$ & \multirow{2}{*}{1.31} & \multirow{2}{*}{0.25} \\
\hline & & No & 193 & (94.6\%) & 385 & (92.1\%) & & \\
\hline
\end{tabular}

${ }^{*} \mathrm{p}<0.05 / 2=0.025$.

Table 14. Gender differences between groups in terms of awareness of the positive effects of coffee on health.

\begin{tabular}{|c|c|c|c|c|c|c|c|c|}
\hline & & & \multicolumn{2}{|c|}{ Consumption } & \multicolumn{2}{|c|}{ Nonconsumption } & \multirow{2}{*}{$\chi^{2}$} & \multirow{2}{*}{$\mathrm{p}$} \\
\hline & & & $\mathrm{n}$ & $\%$ & $\mathrm{n}$ & $\%$ & & \\
\hline \multirow{10}{*}{ Males } & \multirow{2}{*}{ 1. Diabetes mellitus prevention } & Yes & 3 & $(1.0 \%)$ & 2 & $(0.7 \%)$ & \multirow{2}{*}{0.17} & \multirow{2}{*}{0.68} \\
\hline & & No & 285 & (99.0\%) & 277 & $(99.3 \%)$ & & \\
\hline & \multirow{2}{*}{ 2. Cancer prevention } & Yes & 4 & $(1.4 \%)$ & 8 & $(2.9 \%)$ & \multirow{2}{*}{1.50} & \multirow{2}{*}{0.22} \\
\hline & & No & 284 & $(98.6 \%)$ & 271 & $(97.1 \%)$ & & \\
\hline & \multirow{2}{*}{ 3. Diet } & Yes & 7 & $(2.4 \%)$ & 5 & $(1.8 \%)$ & \multirow{2}{*}{0.28} & \multirow{2}{*}{0.60} \\
\hline & & No & 281 & $(97.6 \%)$ & 274 & $(98.2 \%)$ & & \\
\hline & \multirow{2}{*}{ 4. Metabolic syndrome prevention } & Yes & 5 & $(1.7 \%)$ & 3 & $(1.1 \%)$ & \multirow{2}{*}{0.45} & \multirow{2}{*}{0.51} \\
\hline & & No & 283 & $(98.3 \%)$ & 276 & $(98.9 \%)$ & & \\
\hline & \multirow{2}{*}{ 5. Resolution of stress } & Yes & 31 & $(10.8 \%)$ & 32 & $(11.5 \%)$ & \multirow{2}{*}{0.07} & \multirow{2}{*}{0.79} \\
\hline & & No & 257 & $(89.2 \%)$ & 247 & $(88.5 \%)$ & & \\
\hline \multirow{10}{*}{ Females } & \multirow{2}{*}{ 1. Diabetes mellitus prevention } & Yes & 2 & $(1.0 \%)$ & 2 & $(0.5 \%)$ & \multirow{2}{*}{0.54} & \multirow{2}{*}{0.46} \\
\hline & & No & 202 & $(99.0 \%)$ & 416 & $(99.5 \%)$ & & \\
\hline & \multirow{2}{*}{ 2. Cancer prevention } & Yes & 5 & $(2.5 \%)$ & 13 & $(3.1 \%)$ & \multirow{2}{*}{0.21} & \multirow{2}{*}{0.65} \\
\hline & & No & 199 & $(97.5 \%)$ & 405 & (96.9\%) & & \\
\hline & \multirow{2}{*}{ 3. Diet } & Yes & 4 & $(2.0 \%)$ & 23 & $(5.5 \%)$ & \multirow{2}{*}{4.14} & \multirow{2}{*}{0.04} \\
\hline & & No & 200 & $(98.0 \%)$ & 395 & (94.5\%) & & \\
\hline & \multirow{2}{*}{ 4. Metabolic syndrome prevention } & Yes & 1 & $(0.5 \%)$ & 7 & $(1.7 \%)$ & \multirow{2}{*}{1.52} & \multirow{2}{*}{0.22} \\
\hline & & No & 203 & (99.5\%) & 411 & (98.3\%) & & \\
\hline & \multirow{2}{*}{ 5. Resolution of stress } & Yes & 19 & $(9.3 \%)$ & 27 & $(6.5 \%)$ & 163 & 020 \\
\hline & & No & 185 & (90.7\%) & 391 & (93.5\%) & 1.05 & 0.20 \\
\hline
\end{tabular}

tion groups. In short, young females may choose to drink tea rather than coffee more often than young males. In contrast, Yamamoto et al. [17] reported that 68\% female students responded that they like coffee. In this study, only approximately 50\% female respondents considered coffee to be delicious, even in the coffee consumption group. These results may indicate that young females may not drink coffee regularly, although they may like it. Further studies are required to examine the relationship between coffee consumption and preference for the taste of coffee.

In the coffee consumption group, approximately 50\% respondents reported that the reason they liked to drink coffee was because it helped them wake up or because they liked its taste. Caffeine in coffee has a stimulating effect on the central nervous system [18]. Many people who drink coffee expect to feel awake. To consume the same food and drinks regularly, it is very important that a person considers them delicious. Many respondents of both genders in this study drank coffee for its taste. In addition, almost all respondents (approximately 80\%) knew that coffee contains caffeine, but few knew about for the other ingredients in coffee (4.9\% - 5.4\%).

More females (64.8\%) than males (39.4\%) in the coffee nonconsumption group reported that they disliked the taste. Maruyama et al. [19] examined gender differences 
Table 15. Gender differences between groups in terms of awareness of the negative effects of coffee on the body.

\begin{tabular}{|c|c|c|c|c|c|c|c|c|}
\hline & & & \multicolumn{2}{|c|}{ Consumption } & \multicolumn{2}{|c|}{ Nonconsumption } & \multirow{2}{*}{$\chi^{2}$} & \multirow{2}{*}{$\mathrm{p}$} \\
\hline & & & $\mathrm{n}$ & $\%$ & $\mathrm{n}$ & $\%$ & & \\
\hline \multirow{14}{*}{ Males } & \multirow{2}{*}{ 1. Unknown } & Yes & 121 & $(42.0 \%)$ & 173 & $(62.0 \%)$ & \multirow{2}{*}{$22.7^{*}$} & \multirow{2}{*}{0.000} \\
\hline & & No & 167 & $(58.0 \%)$ & 106 & $(38.0 \%)$ & & \\
\hline & \multirow{2}{*}{ 2. Stomach problems } & Yes & 70 & $(24.3 \%)$ & 43 & $(15.4 \%)$ & \multirow{2}{*}{7.02} & \multirow{2}{*}{0.008} \\
\hline & & No & 218 & (75.7\%) & 236 & (84.6\%) & & \\
\hline & \multirow{2}{*}{ 3. Close to a restroom } & Yes & 106 & $(36.8 \%)$ & 58 & $(20.8 \%)$ & \multirow{2}{*}{$17.7^{*}$} & \multirow{2}{*}{0.000} \\
\hline & & No & 182 & $(63.2 \%)$ & 221 & $(79.2 \%)$ & & \\
\hline & \multirow{2}{*}{ 4. Become to loose stools } & Yes & 30 & $(10.4 \%)$ & 19 & $(6.8 \%)$ & \multirow{2}{*}{2.34} & \multirow{2}{*}{0.127} \\
\hline & & No & 258 & $(89.6 \%)$ & 260 & $(93.2 \%)$ & & \\
\hline & \multirow{2}{*}{ 5. Dehydration } & Yes & 12 & $(4.2 \%)$ & 2 & $(0.7 \%)$ & \multirow{2}{*}{7.00} & \multirow{2}{*}{0.008} \\
\hline & & No & 276 & $(95.8 \%)$ & 277 & $(99.3 \%)$ & & \\
\hline & \multirow{2}{*}{ 6. Trembling hands } & Yes & 8 & $(2.8 \%)$ & 4 & $(1.4 \%)$ & \multirow{2}{*}{1.24} & \multirow{2}{*}{0.266} \\
\hline & & No & 280 & $(97.2 \%)$ & 275 & (98.6\%) & & \\
\hline & \multirow{2}{*}{ 7. Getting palpitations } & Yes & 15 & $(5.2 \%)$ & 4 & $(1.4 \%)$ & \multirow{2}{*}{6.23} & \multirow{2}{*}{0.013} \\
\hline & & No & 273 & (94.8\%) & 275 & $(98.6 \%)$ & & \\
\hline \multirow{14}{*}{ Females } & \multirow{2}{*}{ 1. Unknown } & Yes & 74 & $(36.3 \%)$ & 234 & $(56.0 \%)$ & \multirow{2}{*}{$21.3^{*}$} & \multirow{2}{*}{0.000} \\
\hline & & No & 130 & $(63.7 \%)$ & 184 & $(44.0 \%)$ & & \\
\hline & 2 Stomach problems & Yes & 57 & $(27.9 \%)$ & 66 & $(15.8 \%)$ & $128^{*}$ & 0000 \\
\hline & 2. Stomacil piovients & No & 147 & $(72.1 \%)$ & 352 & $(84.2 \%)$ & & 0.000 \\
\hline & 3 Close to a restroom & Yes & 83 & $(40.7 \%)$ & 97 & $(23.2 \%)$ & $204^{*}$ & 0000 \\
\hline & J. Close to d restroum & No & 121 & $(59.3 \%)$ & 321 & (76.8\%) & 20.4 & 0.000 \\
\hline & 4 Berome to loose stools & Yes & 27 & (13.2\%) & 30 & $(7.2 \%)$ & 605 & 0014 \\
\hline & & No & 177 & (86.8\%) & 388 & (92.8\%) & & \\
\hline & 5 Dehvdration & Yes & 8 & (3.9\%) & 9 & $(2.2 \%)$ & 161 & 0204 \\
\hline & & No & 196 & (96.1\%) & 409 & $(97.8 \%)$ & & 0.204 \\
\hline & C Trombling honds & Yes & 4 & (2.0\%) & 7 & (1.7\%) & 07 & 0700 \\
\hline & o. Themoming hallus & No & 200 & (98.0\%) & 411 & $(98.3 \%)$ & 0.07 & 0.199 \\
\hline & 7 Getting palpitations & Yes & 2 & $(1.0 \%)$ & 6 & $(1.4 \%)$ & 0.22 & 0636 \\
\hline & & No & 202 & (99.0\%) & 412 & (98.6\%) & & \\
\hline
\end{tabular}

in taste preference in a group of junior high school students, and reported that taste differs between males and females. Most youth have tasted coffee at least once in their lives. Hence, more females may dislike the taste of coffee based on their past experience, and thus do not choose to drink. In addition, almost all respondents of both genders $(70 \%)$ in the nonconsumption group knew that coffee contains caffeine, but few knew about the other ingredients in coffee. According to Du et al. [13], knowledge and attitude regarding nutrients differ between males and females. However, young people who do not drink coffee may not be interested in it, regardless of gender.

No gender differences were found in responses to the items regarding the effects of coffee on health. More than 80\% respondents expected no adverse effects, particularly in the coffee consumption group. In Japan, it is widely known that tea drinking has healthy effects. According to Matsui [20], about 50\% of nonsugar tea drink- ers found its health benefits very appealing and hence have chosen this beverage. In Japan, coffee is a luxury drink, unlike refreshing drinks such as tea. Approximately $50 \%$ respondents in the coffee consumption group drank coffee because they liked its taste. Thus, people who drink coffee regularly may do so because they enjoy its taste and expect to experience its positive effects, such as helping them to feel awake.

No intergroup gender differences were found in the responses to items regarding the positive and negative effects of coffee on health. More than $80 \%$ of respondents in the coffee consumption group and $40 \%-50 \%$ of those in the coffee nonconsumption group were unaware of these effects. Because coffee has been part of human diet for more than 1000 years [1], many researchers have studied its effects on health. The benefits of coffee for humans have been disputed [21]. However, the present results suggested that the effects of coffee on health are not widely known to young people. Because most respondents 
in the consumption group and more than $65 \%$ of those in the nonconsumption group knew that coffee contains caffeine, it is considered that the caffeine is a widely known component among young people. However, no significant gender differences between the consumption and nonconsumption groups were found regarding the effects of coffee on health. In Japan, alternative healthy drinks such as water or lactic acid bacteria beverages have been manufactured. In addition, many people recognize the healthy effect of tea [20].

On the other hand, the risk of myocardial infarction and glaucoma are increased by coffee consumption [22, 23]. However, regarding the adverse effects of coffee on the body, both male and female respondents in the nonconsumption group ( $>50 \%$ ) were unaware of these effects, and approximately $40 \%$ of those in the consumption group were unaware of these effects. In addition, approximately $40 \%$ of those in the consumption group felt the need to be close to a restroom after drinking coffee. Many studies have reported results of tests of coffee from the medical and pharmaceutical viewpoints [3-7]. The media also frequently reports the effects of coffee. However, the adverse effects of coffee consumption may not be adequately reported. Therefore, young people may know little about these effects regardless of their preference for coffee. It is necessary to spread awareness regarding the positive and negative effects of coffee on health, regardless of coffee consumption. Similar to the coffee-drinking habit of westerners, many Japanese, including the youth, tend to drink coffee during business or social meetings. Moreover, coffee can be freely ordered at a coffee-specialty store, tea shop, or fast food center and can be easily bought at a supermarket or from vending machines. Such circumstances are responsible for giving rise to many coffee drinkers.

\section{Conclusion}

In conclusion, in this study, young males reported drinking more coffee than young females. Among coffee nonconsumers, females disliked the taste of coffee more often than males. Many young people know that coffee contains caffeine, but few know about other components of this beverage. In addition, few people know the positive and negative effects of coffee on health.

\section{REFERENCES}

[1] B. Schilter, C. Cavin, A. Tritscher and A. Constable, "Health Effects and Safety Consideration," In R. J. Clarke and O. G. Vitzthum, Eds., Coffee Recent Developments, Blackwell Science Company, Ames, 2001, p. 166.

[2] T. Yamato, M. Kino, T. Obata, H. Ohta and M. Aomine, "Modulatory Effect of Coffee on Restrained Stress-Induced Release of Neurotransmitters in Rat," Journal of
Japanese Society of Nutrition and Food Science, Vol. 55, No. 2, 2002, pp. 85-91. doi:10.4327/jsnfs.55.85

[3] E. Giovannucci, "Meta-Analysis of Coffee Consumption and Risk of Colorectal Cancer," American Journal of Epidemiology, Vol. 147, No. 11, 1998, pp. 1043-1052. doi:10.1093/oxfordjournals.aje.a009398

[4] A. Tverdal and S. Skurtveit, "Coffee Intake and Mortality from Liver Cirrhosis,” Annals of Epidemiology, Vol. 13, No. 6, 2003, pp. 419-423. doi:10.1016/S1047-2797(02)00462-3

[5] T. Yamaji, T. Mizoue, S. Tabata, S. Ogawa, K. Yamaguchi, E. Shimizu, M. Mineshita and S. Kono, "Coffee Consumption and Glucose Tolerance Status in MiddleAged Japanese Men,” Diabetologia, Vol. 47, No. 12, 2004, pp. 2145-2151. doi:10.1007/s00125-004-1590-5

[6] C. E. Ruhl and J. E. Everhart, “Coffee and Tea Consumption Are Associated with a Lower Incidence of Chronic Liver Disease in the United States," Gastroenterology, Vol. 129, No. 6, 2005, pp. 1928-1936.

doi:10.1053/j.gastro.2005.08.056

[7] M. Inoue, I. Yoshimi, T. Sobue, S. Tsugane and J.P.H.C. Study Group, "Influence of Coffee Drinking on Subsequent Risk of Hepatocellular of Coffee Drinking on Subsequent Risk of Hepatocellular Carcinoma: A Prospective Study in Japan,” Journal of the National Cancer Institute, Vol. 97, No. 4, 2005, pp. 293-300.

doi:10.1093/jnci/dji040

[8] L. F. Andersen, D. R. Jacobs Jr., M. H. Carlsen and R. Blomhoff, "Consumption of Coffee Is Associated with Reduced Risk of Death Attributed to Inflammatory and Cardiovascular Diseases in the Iowa Women's Health Study," American Journal of Clinical Nutrition, Vol. 83, No. 5, 2006, pp. 1039-1046.

[9] H. Iso, C. Date, K. Wakai, M. Fukui, A. Tamakoshi and J.A.C.C. Study Group, "The Relationship between Green Tea and Total Caffeine Intake and Risk for Self-Reported Type 2 Diabetes among Japanese Adults," Annals of Internal Medicine, Vol. 144, No. 8, 2006, pp. 554-562. doi:10.7326/0003-4819-144-8-200604180-00005

[10] M.Garzaro, L. Raimondo, G. Pecorari, M. Sensini, G. Riva, A. Palmo and C. Giordano, "Digestibility, Palatability and Emotional Status after Ingestion of an Iced Dessert: Analysis of Subjective Responses in 100 Healthy Volunteers," Journal of Biological Regulators \& Homeostatic Agents, Vol. 25, No. 1, 2011, pp. 101-107.

[11] K. Yamazawa, K. Hirokawa and H. Shimizu, "Sex Differences in Preferences for Coffee Sweetness among Japanese Students,” Perceptual and Motor Skills, Vol. 105, No. 2, 2007, pp. 403-404.

[12] P. Mirmiran, F. Mohammadi-Nasrabadi, N. Omidvar, F. Hosseini-Esfahani, H. Hamayeli-Mehrabani, Y. Mehrabi and F. Azizi, "Nutritional Knowledge, Attitude and Practice of Tehranian Adults and Their Relation to Serum Lipid and Lipoproteins: Tehran Lipid and Glucose Study," Annals of Nutrition \& Metabolism, Vol. 56, No. 3, 2010, pp. 233-240. doi:10.1159/000288313

[13] W. Du, J. Fu, C. Su, Q. Zhang, F. Zhai and B. Zhang, "Surveys on the Nutrition Literacy of 802 Adults in Jiangxi Province,” Wei Sheng Yan Jiu, Vol. 39, No. 6, 2010, 
pp. 735-738.

[14] M. Izumi and M. Takaya,” Influence of Various of Extraction Conditions and Amount of Chlorogenic Acid on the Taste of Coffee," Journal of Cookery Science of Japan, Vol. 41, No. 4, 2008, pp. 257-261.

[15] A.Drewnowski, S.A. Henderson, A. Levine and C. Hann, "Taste and Food Preferences as Predictors of Dietary Practices in Young Women," Public Health Nutrition, Vol. 2, No. 4, 1999, pp. 513-519.

[16] E. M. Rodenburg, M. Eijgelsheim, J. M. Geleijnse, N. Amin, C. M. van Duijn, A. Hofman, A. G. Uitterlinden, B. H. Stricker and L. E. Visser, "CYP1A2 and Coffee Intake and the Modifying Effect of Sex, Age, and Smoking," American Journal of Clinical Nutrition, Vol. 96, No. 1, 2012, pp. 182-187. doi:10.3945/ajcn.111.027102

[17] T. Yamato, M. Aomine, T. Koga and H. Ohta, "Relationship between Coffee Drinking and Reduction of Mental Stress in Young Women," Food Science and Technology Research, Vol. 11, No. 4, 2005, pp. 395-399. doi:10.3136/fstr.11.395

[18] B. B. Fredholm, "On the Mechanism of Action of Theophylline and Caffeine,” Acta Medica Scandinavica, Vol. 217, No. 2, 1985, pp. 149-153.

[19] S. Maruyama, T. Koda and T. Takahashi, "Gender Dif- ferences among Junior High School Students Regarding Taste Preference of Dried Bonito Soup Stocks,” Japanese Journal of Adolescentology, Vol. 28, No. 1, 2010, pp. 170-176.

[20] Y. Matsui, “The Attractive Power of Oolong Tea,” Journal for the Integrated Study of Dietary Habits, Vol. 11, No. 1, 2000, pp. 2-15. doi:10.2740/jisdh.11.2

[21] H. S. Seo, M. Hirano, J. Shibato, R. Rakwal, I. K. Hwang and Y. Masuo, "Effects of Coffee Bean Aroma on the Rat Brain Stressed by Sleep Deprivation: A Selected Transcript- and 2D Gel-Based Proteome Analysis," Journal of Agricultural and Food Chemistry, Vol. 56, No. 12, 2008, pp. 4665-4673. doi:10.1021/jf8001137

[22] M. C. Cornelis, A. El-Sohemy, E. K. Kabagambe and H. Campos, "Coffee, CYP1A2 Genotype, and Risk of Myocardial Infarction," The Journal of the American Medical Association, Vol. 295, No. 10, 2006, pp. 1135-1141. doi:10.1001/jama.295.10.1135

[23] L. R. Pasquale, J. L. Wiggs, W. C. Willett and J. H. Kang, "The Relationship between Caffeine and Coffee Consumption and Exfoliation Glaucoma or Glaucoma Suspect: A Prospective Study in Two Cohorts," Investigative Ophthalmology \& Visual Science, Vol. 53, No. 10, 2012, pp. 6427-6433. doi:10.1167/iovs.12-10085 